\title{
COSS-Register und Biomaterialbank - Herausforderungen und Chancen
}

Osteosarkome sind die häufigsten primären malignen Knochentumoren bei Kindern, Jugendlichen und jungen Erwachsenen. Seit Gründung der Cooperativen Osteosarkom Studiengruppe COSS im Jahr 1977 werden Patienten mit Osteosarkomen und ähnlichen Knochentumoren aus Deutschland, Österreich und der Schweiz, später auch aus Tschechien und Ungarn, in der COSSDatenbank registriert und zeitlich unbegrenzt nachbeobachtet. Es handelt sich mit über 5000 registrierten Patienten (Stand 10/2020) um eine der größten Osteosarkom-Kohorten weltweit. Die Prognose von hochmalignen lokalisierten Osteosarkomen hat sich in den letzten mehr als 30 Jahren nicht wesentlich verändert [1].

Seit 2005 erfolgt die Datenspeicherung von Patienten, die nicht an einer Therapieoptimierungsstudie von coSs teilnehmen, in einem eigenen Register. Nach Ende der EURAMOS-1-Studie für Patienten bis 40 Jahre und des EURO-B.O.S.S.-Protokolls für Patienten über 40 Jahre werden aktuell alle gemeldeten Patienten in dieses Register rekrutiert.

Veränderte Regularien (EU-Datenschutzgrundverordnung [EU-DSGVO] [2]) und neue Kooperationen machen die Erstellung eines neuen Registerprotokolls notwendig. Bei dem geplanten „Register für Kinder, Jugendliche und Erwachsene mit Osteosarkomen und biologisch verwandten Knochensarkomen: COSS-Register" handelt es sich um ein prospektives, multizentrisches, klinisch/epidemiologisches, nicht-interventionelles und internationales Register. Am COSS-Register teilnehmen können Behandlungszentren mit Sitz in Deutschland, Österreich, Schweiz, Tschechien und Ungarn, vorausgesetzt, dass ein Ethikvotum vorliegt und die Bestimmungen des Protokolls und der EU-DSGVO sowie der jeweils gültigen nationalen Datenschutzgesetze eingehalten werden. Der Start des Registers ist für das 1. Quartal 2021 mit einer unbefristeten Laufzeit geplant.

In das COSS-Register können Patienten jeden Alters, Geschlechts und Tumorstadiums unabhängig von der Teilnahme an einer klinischen Studie eingeschlossen werden, sofern die Diagnose eines Osteosarkoms (jeder Subtyp oder eine Sonderform) oder eines der folgenden Knochensarkome histopathologisch gesichert wurde: undifferenziertes pleomorphes Sarkom, dedifferenziertes oder mesenchymales Chondrosarkom, Fibrosarkom, Angiosarkom bzw. Leiomyosarkom [3]. Zudem muss das Einverständnis zur Teilnahme am Register und an der Biomaterialbank vorliegen.

Im neuen COSS-Register werden weiterhin Daten zu Diagnose, Behandlung und Krankheitsverlauf von Patienten mit Osteosarkomen und verwandten Knochentumoren erfasst. Mit seiner unbefristeten Laufzeit gewährleistet es die Langzeitnachverfolgung dieser Patienten. Ziele des COSS-Registers sind die Erforschung von
Krankheitsursachen sowie die Identifikation von Risikofaktoren für diese Tumoren. Es dient als Rekrutierungsrahmen für zukünftige klinische Phase-I-, Phase-II- oder Therapie-Optimierungsstudien und soll die Datenbasis für zukünftige klinische sowie translationale Forschung erweitern. Ein neues und wichtiges Ziel des Registers ist die Sammlung von biologischem Tumormaterial in einer Biomaterialbank (BMB) für zukünftige Forschungsprojekte.

Die Neufassung des COSS-Registers wird durch verschiedene Hindernisse erschwert: allen voran die gültigen Datenschutzbestimmungen, die eine Trennung von personenbezogenen Daten und medizinischen Forschungsdaten fordern und einen Datenaustausch zwischen behandelnder Klinik und Register sowie BMB bzw. zwischen Register und BMB nur in pseudonymisierter Form gestatten.

Eine weitere Hürde ist die im Vergleich zu einer klinischen Studie meist geringere Bereitschaft von teilnehmenden Kliniken, Patientendaten (zeitnah) in einem Register zu dokumentieren. Im Rahmen eines Registers ist es zudem schwieriger, die Registerteilnehmer zur Einsendung von Biomaterialproben in die BMB zu motivieren. Zur Überwindung dieser beiden Hürden ist die Integration des wissenschaftlichen Begleitprojekts „Tumorprädispositionssyndrome bei Kindern, Jugendlichen und Erwachsenen “ in das COSS-Register geplant. Eine 
Reihe von genetisch determinierten Tumorprädispositionssyndromen (z.B. Li-Fraumeni-Syndrom, familiäres Retinoblastom, Rothmund-Thomson-Syndrom) gehen mit einem erhöhten Osteosarkomrisiko einher [4]. Pathogene Keimbahnvariationen werden für das Osteosarkom in einer Häufigkeit von $6-10 \%$ berichtet [5]. Eine aktuelle Studie konnte bei mehr als einem Viertel der untersuchten Osteosarkompatienten eine pathogene oder wahrscheinlich pathogene Genvariante nachweisen [6]. Ziel des Projektes ist die prospektive Erfassung der Häufigkeit und Art genetischer Keimbahnmutation bei Kindern, Jugendlichen und jungen $\mathrm{Er}$ wachsenen mit Osteosarkom. Zusätzlich soll der Zusammenhang zwischen Keimbahnveränderungen und dem Manifestationsalter und zu bekannten prognostischen Faktoren wie dem Ansprechen auf die Chemotherapie erforscht werden.

Neben Keimbahnveränderungen rücken auch somatische Genveränderungen hinsichtlich ihrer Bedeutung für die Entstehung und Behandlung von Osteosarkomen zunehmend in den wissenschaftlichen Fokus. Bislang fehlt eine entitätsspezifische BMB in Deutschland, die eine systematische Erforschung von Osteosarkomen auf zellulärer und molekularer Ebene ermöglicht.

In Ergänzung zum COSS-Register in Stuttgart soll daher am Klinikum Kassel eine korrespondierende Proben-Datenbank für diese Erkrankung etabliert werden. Die medizinischen Daten des COSS-Registers in Stuttgart werden mittels Zentralem Datenmanagement der Gesellschaft für pädiatrische Onkologie und Hämatologie (GPOH) mit den Proben bzw. Probenbegleitdaten der Proben-Datenbank am Institut für Pathologie am Klinikum Kassel datenschutzkonform verknüpft.

Die BMB soll als entitätsspezifische BMB der $\mathrm{GPOH}$ etabliert werden, welche einheitlich die strukturellen, organisatorischen und qualitativen Anforderungen an pädiatrisch hämato-/onkologische Biomaterialbanken in Deutschland vorgibt.

Die BMB für Osteosarkome soll - zunächst auf nationaler Ebene - Gewebe- und Blutproben von primären und sekundären Neuerkrankungen aller Altersstufen und Stadien sammeln. Dabei sollen prätherapeutisch, präoperativ, nach Therapieabschluss und ggf. im Rezidiv schockgefrorenes und paraffiniertes Restgewebe bzw. Blutproben für genetische Analysen, Proteinanalysen und Analysen zirkulierender, zellfreier TumorDNA (ccf-DNA) asserviert werden. Blutproben werden im Rahmen des geplanten wissenschaftlichen Projekts vor der Asservierung auf bekannte Keimbahnmutationen untersucht. Ein wichtiges Anliegen der BMB ist, molekular gut definierte Kollektive für weitere Forschungsvorhaben verfügbar machen zu können.

Zusammenfassend sollen das neue COSSRegister und die geplante BMB als technische Plattform zur Identifikation neuer prognostischer und prädiktiver Marker sowie therapeutischer Zielstrukturen gemeinsam langfristig ermöglichen, die Prognose von Osteosarkompatienten und die Therapieverträglichkeit zu verbessern.

\section{Interessenkonflikt}

SHN hat innerhalb der letzten 3 Jahre Forschungsunterstützung von der Fa. EISAI erhalten.

\section{Autorinnen/Autoren}

Stefanie Hecker-Nolting ${ }^{1}$, Sandra Gottschling ${ }^{2}$, Michaela Nathrath $^{3}$

${ }^{1}$ Cooperative Osteosarkom Studiengruppe COSS, Zentrum für Kinder- Jugend- und Frauenmedizin, Pädiatrie 5 (Onkologie,
Hämatologie, Immunologie), Klinikum Stuttgart - Olgahospital

${ }^{2}$ Klinik für Onkologie und Hämatologie, Klinikum Kassel

${ }^{3}$ Klinik für Pädiatrische Hämato-Onkologie, Psychosomatik und Systemerkrankungen, Klinikum Kassel

\section{Korrespondenzadresse}

\section{Dr. Stefanie Hecker-Nolting}

Cooperative Osteosarkom Studiengruppe COSS, Zentrum für Kinder- Jugend- und Frauenmedizin, Pädiatrie 5 (Onkologie, Hämatologie, Immunologie), Klinikum Stuttgart - Olgahospital, Germany

E-Mail: s.hecker-nolting@klinikum-stuttgart.de

\section{Literatur}

[1] Isakoff MS, Bielack SS, Meltzer P et al. Osteosarcoma: Current Treatment and a Collaborative Pathway to Success. J Clin Oncol 2015; 33 (27): 3029-35. doi: 10.1200/JCO.2014.59.4895

[2] Verordnung (EU) 2016/679 des Europäischen Parlaments und des Rates vom 27. April 2016 zum Schutz natürlicher Personen bei der Verarbeitung personenbezogener Daten, zum freien Datenverkehr und zur Aufhebung der Richtlinie 95/46/EG (Datenschutz-Grundverordnung).

[3] WHO Classification of Tumours Editorial Board: Soft Tissue and Bone Tumours, Lyon (France) International Agency for Research on Cancer; 2020 (WHO Classification of Tumours series, 5th ed; vol. 3) https://publications.iarc.fr/588

[4] Mirabello L, Yeager M, Mai PL et al. Germline TP53 variants and susceptibility to osteosarcoma. J Natl Cancer Inst 2015; 107: djv101. doi: 10.1093/jnci/djv101

[5] Gianferante DM, Mirabello L, Savage SA et al. Germline and somatic genetics of osteosarcoma - Connecting aetiology, biology, and therapy. Nat Rev Endocrinol 2017; 13: $480-$ 491. doi: $10.1038 /$ nrendo.2017.16

[6] Mirabello L, Zhu B, Koster R et al. Frequency of pathogenic germline variants in cancersusceptibility genes in patients with osteosarcoma. JAMA Oncol 2020; 6: 724-734. doi: 10.1001/jamaoncol.2020.0197 